\title{
Bericht AOTrauma-Reisestipendium 2017
}

Arne Berner

\section{Die AOTrauma Deutschland vergibt jährlich mindestens ein Reisestipendium zur Förderung des Erfahrungsaustausches und der Weiterbildung bei angesehenen Experten in unserer Disziplin. Im folgenden Artikel berichtet PD Dr. Arne Berner, Charité Berlin, von seinen Erfahrungen.}

Mit Unterstützung durch die AOTrauma Deutschland war es mir möglich, Prof. Ted Miclau in San Francisco im Zuckerberg General Hospital and Trauma Center zu besuchen. Das Krankenhaus ist das größte Trauma-Zentrum in der San Francisco Bay Area und versorgt viele Polytraumen.

Das gesamte Team um Prof. Miclau hat mich sehr nett aufgenommen und in die Abläufe integriert. Um 7 Uhr ging es mit der Morgenbesprechung los, in der die Fälle aus der Nacht sowie kurzfristige Änderungen des OPPlans besprochen wurden. Im Anschluss danach wurden entweder die Post-OP-Bilder der letzten Woche besprochen oder der OP-Plan der nächsten Woche. An zwei weiteren Tagen gab es jeweils eine Fortbildung für die Residents mit unterschiedlichen Themen. Es war jederzeit möglich, Fragen zu stellen, was auch von vielen Residents genutzt wurde. Es wurde sich insgesamt sehr viel Zeit für Aus- und Weiterbildung der Ärzte genommen.

Gegen 8:30 Uhr wurde die 1. Operation gestartet. Das Bemerkenswerte hieran war, das immer einer der jüngeren Residents den Zugang machen durfte, wobei ihm von einem erfahrenen Resident oder einem der Fellows assistiert wurde. Im Laufe der OP kam dann der Attending dazu und es wurden den jüngeren Kollegen weitere Schritte assistiert. Hierdurch dauerte die gesamte OP natürlich länger, dies schien jedoch keinen wirklich zu stören, und die Operationen wurden alle sehr ruhig bis zum Ende assistiert. Trotz dieser intensiven Ausbildung waren die postoperativen Ergebnisse sehr gut. Die personelle und auch materielle Ausstattung im OP war im Vergleich zu Deutschland ausgezeichnet. In jedem Saal, in dem Röntgen notwendig war, gab es extra hierfür einen extra Röntgenassistenten, der ausschließlich den Bildwandler bediente. Weiterhin war meist von jeder Firma ein Vertreter anwesend, deren Implantate bei der jeweiligen OP benutzt wurden.

Direkt angegliedert an die Klinik war eine Forschungsabteilung, die sich intensiv mit dem Thema „Bone regen-

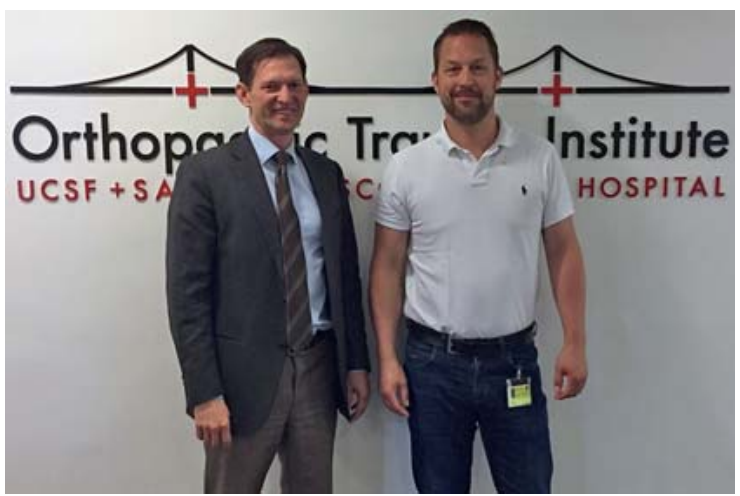

Abb. 1 Ted Miclau mit Arne Berner

eration" befasste. Somit gab es auch hier eine intensive Kommunikation zwischen den Wissenschaftlern und den Klinikern.

An meinen letzten beiden Tagen konnte ich an dem International San Francisco Orthopaedic Trauma Course teilnehmen, welcher neben guten Vorträgen auch viele praktische Workshops hatte.

Insgesamt kann man sagen, dass ich durch dieses Fellowship sehr interessante Einblicke in ein anderes Gesundheits- und Ausbildungssystem bekommen habe, die mir sowohl beruflich als auch privat positiv in Erinnerung bleiben. Mein Dank gilt Prof. Miclau und seinem Team sowie der AOTrauma für die finanzielle Unterstützung durch das Reisestipendium.

PD Dr. Arne Berner, Charité Berlin

\section{Bibliografie}

DOI https://doi.org/10.1055/a-0647-2548

OP-JOURNAL 2018; 34: 181 @ Georg Thieme Verlag KG Stuttgart · New York ISSN 0178-1715 